\title{
Analytical method of forecasting the settlement of water-saturated soils over time
}

\author{
Irina Yudina* \\ Moscow State University of Civil Engineering, Yaroslavskoe shosse, 26, Moscow, 129337, Russia
}

\begin{abstract}
The paper deals with the formulation and solution of the consolidation problem for weak water-saturated soil bases by using exponential functions to describe the initial pore water pressure with zero boundary conditions on the surface of the base and at infinity. The solution of this problem was obtained in closed form by the source function method. Expressions are given for the pore water pressure and degree of consolidation, as well as an example of such calculation.
\end{abstract}

\section{Introduction}

The problem of the construction of structures on weak water-saturated clay soils is connected with forecasting the magnitude and compaction speed, due to there interaction with various types of structures.

The experience of structures operation on such soils shows that the value and rate of development of settlement sometimes higher than expected one and it leads to emergency situations. The main factors that determine the development of ground settlements over time are its filtration and rheological properties, as well as the thickness of a densified layer. Depending on the material composition of the weak water-saturated soil, the intensity of the consolidation process is affected either by the filtration or rheological properties of the soil [1], [2]. As a criterion for registration this or another factor, one can consider a dimensionless parameter characterizing the integral toughness of an aggregate of weak soil $\mu=\left(\eta \cdot H^{2}\right) \cdot c_{v}, \eta$ - the creep characteristic; $H$ - the largest size of the considered aggregate; $c_{v}$ - coefficient of consolidation [2]. For soils with a low filtration factor $(\mathrm{k}<10$ $\mathrm{cm} / \mathrm{s}$ ), and for large values of the dimensionless parameter $\mu \geq 10$, the rate of consolidation will be determined mainly by the filtration properties of the soil.

\section{Main Part}

As the analysis of researches shows, weak water-saturated soils belong to this category. In these soils not only a creep of the soil skeleton during the filtration consolidation, but also secondary consolidation are poorly manifested. So, the results of special large-scale tests of

\footnotetext{
* Corresponding author: yudinaim@mail.ru
} 
the fluid consistency loam near Moscow showed that the secondary consolidation in them is negligible and account for several percent. As a result, we can consider the process of filtration consolidation without taking creep of the solid skeleton into account.

Thus, the main task of forecasting the settlements of structures erected on weak soils is connected with solving the problems of filtration consolidation. In such cases the method of the equivalent layer by N.A. Tsytovich is used [3]. At the same time, the idea of an equivalent layer method can also be developed for the case of curvilinear variation of sealing stresses over the depth of a layer on the basis of known solutions, as proposed in this article. A similar method was used earlier in forecasting the rising of the bottom in time [4].

The procedure for calculating the settlements of weak water-saturated grounds over time is as follows. We consider a one-dimensional problem of consolidation of a layer of water-saturated soil under the vertical and horizontal stresses' influence, which vary in depth according to curvilinear laws. The latest are determined from the exact solution in the linear formulation of the problem of the evenly distributed over a rectangular area $(2 l \times 2 b)$ load's action, with value $-q$ on a ground half-space and they are described approximately, by the sum of exponential functions. Thus, the compaction pressure and the sum of the main stresses for the central axis, which vary in depth, have such form:

$$
\begin{aligned}
& \sigma_{Z}=q \sum_{i=1}^{3} a_{i} \exp \left(-d_{i} m\right) \\
& \theta=q(1+\mu) \sum_{i=1}^{2} a_{i} \exp \left(-b_{i} m\right)
\end{aligned}
$$

Where $z$ - the depth of the considered point; $m=z / b, a_{\mathrm{i}}, b_{\mathrm{i}}, d_{\mathrm{i}}-$ the parameters that functionally connected with the ratio of the sides of the loaded area, determined from the expressions:

$$
\begin{aligned}
& a_{1}=0.539 \quad a_{2}=1.471 \quad a_{3}=-1.01 \\
& d_{1}=0.153+0.276 / n-0.075 / n^{2} \\
& d_{2}=1.112-0.78 / n+1.169 / n^{2} \\
& d_{3}=1.896-1.34 / n+2.598 / n^{2} \\
& b_{1}=0.148+0.405 / n+0.075 / n^{2} \\
& b_{2}=0.979-\frac{0.217}{n}+\frac{0.535}{n^{2}}, \text { where } n=\frac{l}{b}
\end{aligned}
$$

For angular points, the formulas take the following forms:

$$
\begin{aligned}
& \sigma_{z}=q \sum_{i=1}^{3} \frac{a_{i}}{4} \exp \left(-d_{i} m\right) \\
& \theta=q(1+\mu) \sum_{i=1}^{2} \frac{a_{i}}{4} \exp \left(-b_{i} m\right)
\end{aligned}
$$

The proposed formulas describe the stress distribution with high accuracy, in addition, they are not cumbersome and are convenient for calculations. 
Having expressions for the sum of the main stresses and the component of the vertical stresses, we obtain expressions for the vertical deformations and settlement from the wellknown formulas:

$$
\begin{aligned}
& \varepsilon_{Z}=\frac{q}{E}(1+\mu)\left[\sum_{i=1}^{3} a_{i} \exp \left(-d_{i} m\right)-\mu \sum_{i=1}^{2} a_{i} \exp \left(-b_{i} m\right)\right] \\
& S=\frac{q b(1+\mu)}{E}\left\{\sum_{i=1}^{3} \frac{a_{i}}{d_{i}}\left[1-\exp \left(-d_{i} \bar{H}\right)\right]-\mu \sum_{i=1}^{2} \frac{a_{i}}{b_{i}}\left[1-\exp \left(-b_{i} \bar{H}\right)\right]\right\}
\end{aligned}
$$

where $\bar{H}=H / b ; H$ - the depth of the compressed width.

With $H \rightarrow \infty$,

$$
S=\frac{q b(1+\mu)}{E}\left(\sum_{i=1}^{3} a_{i} / d_{i}-\mu \sum_{i=1}^{2} a_{i} / b_{i}\right)
$$

To apply the resulting formulas in the case when the base is not homogeneous (multi-layer), we can assume in engineering calculations that the heterogeneity of the base insignificantly affects the stress diagrams. In this case we have:

$$
\begin{aligned}
& S=q b \sum_{j=1}^{n} \frac{1+\mu_{j}}{E_{j}} \cdot\left\{\sum_{i=1}^{3} \frac{a_{i}}{d_{i}}\left[\exp \left(-\overline{d_{i}} h_{j-1}\right)-\exp \left(-\overline{d_{i}} h_{j}\right)\right]-\right. \\
& \left.-\mu_{j} \sum_{i=1}^{2} \frac{a_{i}}{b_{i}}\left[\exp \left(-\overline{b_{i}} h_{j-1}\right)-\exp \left(-\overline{b_{i}} h_{j}\right)\right]\right\}
\end{aligned}
$$

The formulas (2), (3) and (4) take horizontal stresses into account. In the engineering calculation of the structures' settings these stresses are neglected. Then formula of the settlement on multylayered base has this form.

$$
\begin{aligned}
& S=q b \sum_{j=1}^{n} \frac{\beta_{j}}{E_{j}}\left\{\sum_{i=1}^{3} \frac{a_{i}}{d_{i}}\left[\exp \left(-\overline{d_{i}} h_{j-1}\right)-\exp \left(-\overline{d_{i}} h_{j}\right)\right]\right\}= \\
& =q b \sum_{j=1}^{n} m_{v j}\left\{\sum_{i=1}^{3} \frac{a_{i}}{d_{i}}\left[\exp \left(-\overline{d_{i}} h_{j-1}\right)-\exp \left(-\overline{d_{i}} h_{j}\right)\right]\right\}
\end{aligned}
$$

Where $\mu_{j}, E_{j}, m_{v j}$ - corresponding deformation parameters of the $\mathrm{j}$ layer, $\bar{d}_{i}=d_{i} / b$.

To solve the problem of interest, we consider the application of the obtained empirical formulas (2) taking the consolidation processes into account.

Introducing the notion of reduced deformation modules for the initial pore pressure (2), we have: 


$$
P_{w}(z, 0)=\frac{A_{0}}{3} q\left(1+\mu_{n p}\right) \sum_{i=1}^{2} a_{i} \exp \left(-b_{i} m\right)
$$

where $A_{0}$ - initial pore pressure coefficient; without taking horizontal stresses into account:

$$
P_{w}(z, 0)=\frac{A_{0}}{3} q \frac{1+\mu_{n p}}{1-\mu_{n p}} \sum_{i=1}^{3} a_{i} \exp \left(-d_{i} m\right)
$$

The drainage conditions are placed on the free surface of the ground at an infinite distance from it, which corresponds to the actual filtration conditions. Thus, for $P_{w}(0, t)=P_{w}(\infty, t)=0$ using source functions [2] in the form:

$$
P_{w}(z, t)=\frac{1}{2 \sqrt{\pi c_{v} t}} \int_{0}^{\infty} P_{w}(\zeta)\left\{\exp \left[-\frac{(z-\zeta)^{2}}{4 c_{v} t}\right]-\exp \left[-\frac{(z+\zeta)^{2}}{4 c_{v} t}\right]\right\} d \zeta
$$

the consolidation problem has been solved, which leads to a closed expression for the pore pressure, which has this form:

$$
\begin{aligned}
& P_{w}(z, t)=\frac{A_{0} q\left(1+\mu_{\text {пр }}\right)}{3} \sum_{i=1}^{2} a_{i}\left\{\exp \left[-\overline{b_{i}}\left(z-c_{\nu} \overline{b_{i}} t\right)\right] \times\left[\Phi_{i}\left(\frac{z-2 c_{v} \overline{b_{i}} t}{\sqrt{2 c_{\nu} t}}\right)+\frac{1}{2}\right]-\right. \\
& \left.-\exp \left[\overline{b_{i}}\left(z+c_{v} \overline{b_{i}} t\right)\right] \times\left[\Phi_{i}\left(\frac{z+2 c_{v} \overline{b_{i}} t}{\sqrt{2 c_{\nu} t}}\right)-\frac{1}{2}\right]\right\}
\end{aligned}
$$

Without taking horizontal stresses into account:

$$
\begin{aligned}
& P_{w}(z, t)=\frac{A_{0}}{3} q \frac{1+\mu_{\text {пр }}}{1-\mu_{\text {пр }}} \sum_{i=1}^{2} a_{i}\left\{\exp \left[-\overline{d_{i}}\left(z-c_{\nu} \overline{d_{i}} t\right)\right] \times\left[\Phi_{i}\left(\frac{z-2 c_{v} \overline{d_{i}} t}{\sqrt{2 c_{\nu} t}}\right)+\frac{1}{2}\right]-\right. \\
& \left.-\exp \left[\overline{d_{i}}\left(z+c_{v} \overline{d_{i}} t\right)\right] \times\left[\Phi_{i}\left(\frac{z+2 c_{\nu} \overline{d_{i}} t}{\sqrt{2 c_{\nu} t}}\right)-\frac{1}{2}\right]\right\}
\end{aligned}
$$

Where $\Phi_{i}$ - probability integral, $c_{v}$-coefficient of the consolidation, $\overline{b_{i}}=b_{i} / b$.

Having an expression for the distribution of pore pressure, we obtain an expression for the settlements over time:

$$
\begin{aligned}
& S(t)=\frac{1+\mu}{E} \int_{0}^{H}\left[\sigma_{z}-P_{w}(z, t)\right] d z-\frac{\mu}{E} \int_{0}^{H}\left[\theta-3 P_{w}(z, t)\right] d z= \\
& =S(\bar{H})-\left(1-\mu_{n p}\right) m_{v} \int P_{w}(z, t) d z
\end{aligned}
$$

Without taking horizontal stresses into account:

$$
S(t)=\frac{\beta}{E} \int_{0}^{H}\left[\sigma_{z}-P_{w}(z, t)\right] d z=S\left(\overline{H)}-m_{v} \int_{0}^{H} P_{w}(z, t) d z\right.
$$

Where $S(\bar{H})$ - stabilized settlements.

Substituting (10) into (12), (11) into (13) and integrating, we obtain an expression taking horizontal stresses into account: 


$$
\begin{aligned}
& S(t)=\frac{q b(1+\mu)}{E}\left\{\sum_{i=1}^{3} \frac{a_{i}}{d_{i}}\left[1-\exp \left(-\overline{d_{i}} H\right)\right]-\mu \sum_{i=1}^{2} \frac{a_{i}}{b_{i}}\left[1-\exp \left(-\overline{b_{i}} H\right)\right]\right\}- \\
& -\frac{A_{0}\left(1-\mu_{n p}\right)}{3} m_{V} q b \sum_{i=1}^{2} \frac{a_{i}}{b_{i}} \exp \left(c_{v}{\overline{b_{i}}}^{2} t\right)\left\{\exp \left(\overline{b_{i}} H\right) \times\left[\Phi_{i}\left(\frac{H+2 c_{v} \overline{b_{i}} t}{\sqrt{2 c_{v} t}}\right)-\frac{1}{2}\right]-\right. \\
& \left.-\exp \left(-\overline{b_{i}} H\right) \times\left[\Phi_{i}\left(\frac{H-2 c_{v} \overline{b_{i}} t}{\sqrt{2 c_{v} t}}\right)+\frac{1}{2}\right]+\left[1-2 \Phi_{i}\left(\overline{b_{i}} \sqrt{2 c_{v} t}\right)\right]\right\}
\end{aligned}
$$

Without taking horizontal stresses into account:

$$
\begin{aligned}
& S(t)=m_{V} q b\left\{\sum_{i=1}^{3} \frac{a_{i}}{d_{i}}\left[1-\exp \left(-\overline{d_{i}} H\right)\right]-\frac{1+\mu_{n p}}{1-\mu_{n p}} \frac{A_{0}}{3} \cdot \sum_{i=1}^{3} \frac{a_{i}}{d_{i}} \exp \left(c_{v}{\overline{d_{i}}}^{2} t\right) \times\right. \\
& \times\left\{\exp \left(\overline{d_{i}} H\right)\left[\Phi_{i}\left(\frac{H+2 c_{v} \overline{d_{i}} t}{\sqrt{2 c_{v} t}}\right)-\frac{1}{2}\right]-\exp \left(-\overline{d_{i}} H\right)\left[\Phi_{i}\left(\frac{H-2 c_{v} \overline{d_{i}} t}{\sqrt{2 c_{v} t}}\right)+\frac{1}{2}\right]+\right. \\
& \left.\left.+\left[1-2 \Phi_{i}\left(\overline{d_{i}} \sqrt{2 c_{v} t}\right)\right]\right\}\right\}
\end{aligned}
$$

If $t=0$,

$S(t)=m_{V} q b \sum_{i=1}^{3} \frac{a_{i}}{d_{i}}\left[1-\exp \left(-\overline{d_{i}} H\right)\right]-\left(1-\frac{1+\mu_{\text {пр }}}{1-\mu_{\text {пр }}} \cdot \frac{A_{0}}{3}\right)$

If $\mu_{n p}=0,5 ; A_{0}=1$ - which corresponds to the complete water saturation of soil pores $S\left(t_{0}\right)=0$.

For wide application of the obtained results, the corresponding tables have been compiled, which simplify the calculation technique.

To analyze the obtained formulas, the settlements of the ground base over time have been calculated from the action of a massive foundation with the following parameters: the area of the foundation base is $9 \times 6 \mathrm{~m}$, the soil is a homogeneous clay characterized by the coefficient: $m_{V}=6 \cdot 10^{-3} \mathrm{~m}^{2} / N ; \mu=0.4 ; k_{f}=0.15 \mathrm{~cm} / y$; ground pressure is $q=0.25$ $\mathrm{MPa}$.

In this case, using different methods we calculated the values of the stabilized settlements: $3.3 \mathrm{~cm}$ by the method of layer-wise summation; $6.2 \mathrm{~cm}$ by the equivalent layer method; $5.8 \mathrm{~cm} ; 3.4 \mathrm{~cm}$-by the proposed method, with and without taking the horizontal stresses into account. Experimental observations of the object settlements were $5.5 \mathrm{~cm}$ which agrees well with the settlements obtained by the proposed method $(5.8 \mathrm{~cm})$. The nature of the development of settlements over time (Fig. 1) differs significantly from curves 1 and 2, obtained by the equivalent layer method, which have a smooth attenuation. Curve number 3 has two characteristic areas: intensive consolidation $\left(t<t_{1}\right)$ and long-term consolidation $\left(t>t_{1}\right)$. In addition, it is closer to case -2 , which takes two-way water filtration into account (Fig. 1). 


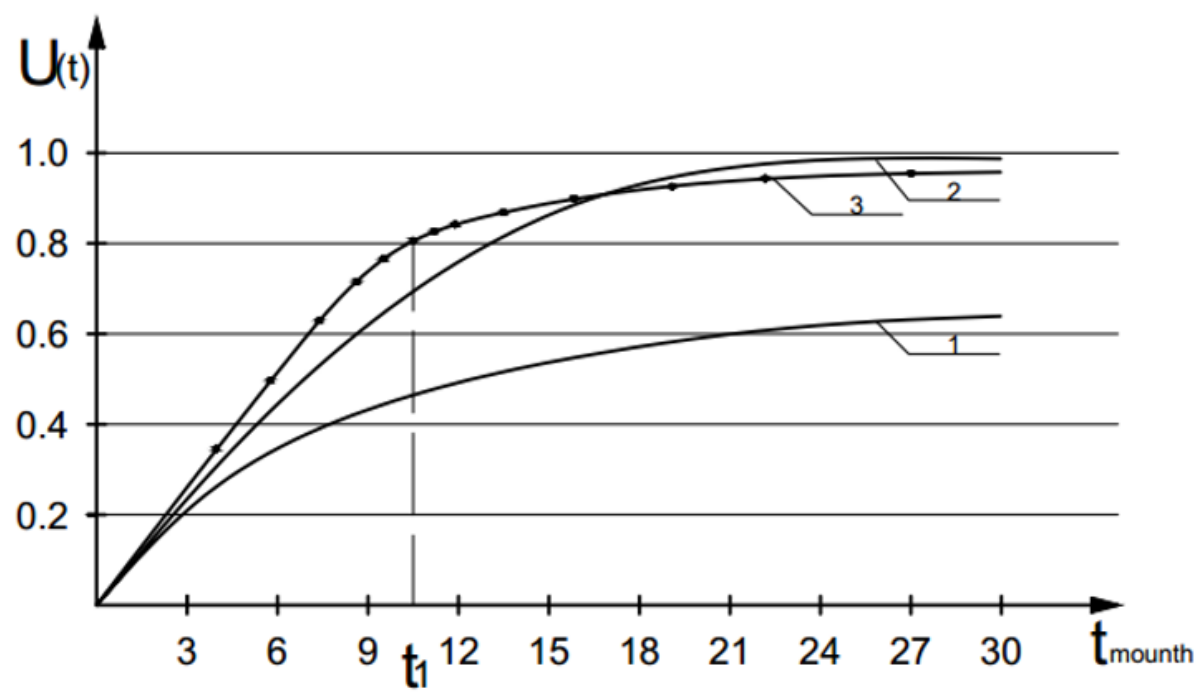

Fig. 1. Dependences of changing of the degree of consolidation over time, calculated 1 and 2 - by the equivalent layer method, respectively with one-sided and two-sided filtration; 3 - by the proposed method

\section{Conclusion}

Thus, the proposed calculation method describes the development of settlements of waterdeposited ground over time quite well and can be used in engineering design practice for their forecasting.

\section{References}

1. Z.G. Ter-Martirosyan, Rheological soil parameters and calculations of bases of construction, 200 (Stroyizdat Publ., Moscow, 1990)

2. Z.G. Ter-Martirosyan, Soil mechanics, 552 (ACU Publ., 2009)

3. N.A. Tsytovich and others, Settlement speed prediction of bases of constructions (Stroyizdat Publ., Moscow, 1967)

4. I.M. Yudina, Decompaction of soils of the basis and its accounting at the sludge forecast (MSUCE, Moscow, 1989) 